\title{
Neutrino Pair Emission from Cooper Pair Breaking and Recombination in Superfluid Quark Matter
}

\author{
Prashanth Jaikumar and Madappa Prakash \\ Department of Physics \& Astronomy, SUNY at Stony Brook, New York 11794-3800
}

(October 25, 2018)

\begin{abstract}
For the low energy Standard Model neutrino-matter interactions, we calculate neutrino pair $(\nu \bar{\nu})$ emissivites in superfluid quark matter. Just below the critical temperature, Cooper pairs continuously break and recombine, resulting in the emission of $\nu \bar{\nu}$ pairs with a rate that greatly exceeds the standard quark modified Urca and bremsstrahlung rates. At the same baryon density in baryonic and quark matter, the ratio of baryon to quark $\nu \bar{\nu}$ emissivites lies in the range $2-5$ for the densities of interest in the long-term cooling of solar mass compact stars. We also find that in matter containing hyperons, $\nu \bar{\nu}$ emission can occur with hyperons of all species.
\end{abstract}

PACS: 97.60.Jd, 24.85.+p, 26.60.+c, 95.30.Cq

In a prescient paper, Flowers, Ruderman, and Sutherland [1] showed that just below the critical temperature $T_{c}$, the neutrino pair $(\nu \bar{\nu})$ emissivity from the breaking and recombination of superfluid neutron pairs $(n+n \rightarrow n+n+\nu+\bar{\nu})$ greatly exceeds that from the so-called modified Urca processes $\left(n+n \rightarrow n+p+e^{-}+\bar{\nu}\right.$ and $\left.n+p \rightarrow n+n+e^{+}+\nu\right)$ in neutron star interiors. It is only recently that the pair breaking and formation $(\mathrm{PBF})$ processes have been included in calculations of the long term cooling of neutron stars [2]. The principal effect of the PBF process is to rapidly cool the star during its early hundreds of years of thermal evolution, until competing $\nu$-emitting processes (for recent reviews see Ref. [3]) begin to dominate the further cooling of the star for several thousands or million years that they might remain observable with such instruments as HST, Chandra, and XMM. The precise extent to which the core temperature falls depends on the smallest of the pairing gaps among the superfluid species present in the star (cf. [1] and references therein). To date, the role of PBF processes has been explored only for baryon superfluidity [2].

In this work, we calculate the $\nu \bar{\nu}$ emissivity in superfluid quark matter likely to be encountered in neutron star interiors [5]. For the low energy Standard Model neutrino-matter interactions, we show that the predominant contribution to the total $\nu \bar{\nu}$ emissivity comes from the vector channel, while the axial channel provides a small correction. This heirarchy is similar to that found in baryonic matter [1]. We also consider the effect of quark masses on the emissivities in limiting cases. We compare our results for $\nu \bar{\nu}$ emissivities with those of the modified Urca and bremsstrahlung processes in both quark and nucleonic matter [6]. Expected $\nu \bar{\nu}$ emissivities from hyperons in baryonic matter and from $p$-wave pairing in quark matter are also addressed. 
The Hamiltonian that describes the neutral current quark-neutrino weak interaction is given by [9]

$$
H_{W}=\frac{G_{F}}{\sqrt{2}}\left[\bar{\psi}^{i, a} \gamma^{\lambda}\left(c_{V}-c_{A} \gamma_{5}\right) \psi_{i, a}\right]\left[\bar{\psi}_{\nu_{\ell}} \gamma_{\lambda}\left(1-\gamma_{5}\right) \psi_{\nu_{\ell}}\right]
$$

here $G_{F}$ is the Fermi constant for the weak interaction, and $c_{V}$ and $c_{A}$ are flavor dependent vector and axial-vector coupling constants, respectively. The index $i$ denotes quark flavor $\left(N_{f}=3\right)$ and $a$ denotes color $\left(N_{c}=3\right)$. The index $\ell$ stands for lepton flavor $e, \mu$ or $\tau$. At leading order (tree level), flavor changing processes do not occur through the weak interaction, hence the diagonal structure in flavor and color space. Unlike baryons, quarks in a superfluid phase can lock themselves in preferred directions in color and flavor space [5]. However, we are considering neutrino pair $(\nu \bar{\nu})$ production mediated by the neutral current weak interaction, which is blind to color and flavor. These degrees of freedom are, however, counted in the density of states at the Fermi surface.

We begin with the case in which quarks of all flavors are taken to be massless. In this case, the full structure of the weak interaction through 4-component Weyl spinors is needed (In the nonrelativistic case, e.g. for baryons in the superfluid phase, the $\mathrm{V}-\mathrm{A}$ interaction has large matrix elements only in the $2 \times 2$ block diagonals in the space of 4 -component spinors. This allows for simpflication in that 2-component spinors with a suitable normalization are adequate.) The helicity basis (which is also the chirality basis for massless particles when we identify positive helicity with right handed particles $\{R\}$ and negative helicity with left handed $\{L\}$ ones) is convenient in this case. The quark field operator may be expressed as 1

$$
\psi(\overrightarrow{\mathbf{x}})=\sum_{\mathbf{p}}\left(e^{-i \overrightarrow{\mathbf{p}} \cdot \overrightarrow{\mathbf{x}}} u_{p}\left\{\chi_{\overrightarrow{\mathbf{p}}, R} b_{\overrightarrow{\mathbf{p}}, R}+\chi_{\overrightarrow{\mathbf{p}}, L} b_{\overrightarrow{\mathbf{p}}, L}\right\}+e^{i \overrightarrow{\mathbf{p}} \cdot \overrightarrow{\mathbf{x}}} v_{p}\left\{\chi_{\overrightarrow{\mathbf{p}}, R} b_{\overrightarrow{\mathbf{p}}, L}^{\dagger}-\chi_{\overrightarrow{\mathbf{p}}, L} b_{\overrightarrow{\mathbf{p}}, R}^{\dagger}\right\}\right),
$$

where $\chi$ 's are 4-component Weyl spinors and we have paired particles of opposite helicity according to

$$
b_{\overrightarrow{\mathbf{p}}, R}=u_{p} a_{\overrightarrow{\mathbf{p}}, R}+v_{p} a_{-\overrightarrow{\mathbf{p}}, L}^{\dagger} \quad \text { and } \quad b_{\overrightarrow{\mathbf{p}}, L}=u_{p} a_{\overrightarrow{\mathbf{p}}, L}-v_{p} a_{-\overrightarrow{\mathbf{p}}, R}^{\dagger} .
$$

The quasiparticle excitations are determined through a Bogoliubov-Valatin transformation on the usual creation and annihilation operators $\left(a\right.$ and $\left.a^{\dagger}\right)$, and are distributed according to the Fermi-Dirac function at finite temperature. For isotropic ${ }^{1} \mathrm{~S}_{0}$ pairing, the coherence factors $u_{p}$ and $v_{p}$ satisfy

$$
u_{p} v_{p}=\frac{\Delta}{2 E_{p}}, \quad u_{p}^{2}=\frac{1}{2}\left(1+\frac{\epsilon_{p}}{E_{p}}\right), \quad \text { and } \quad v_{p}^{2}=\frac{1}{2}\left(1-\frac{\epsilon_{p}}{E_{p}}\right) .
$$

The quasiparticle excitations carry momentum $p$ and energy $E_{p}=\sqrt{\epsilon_{p}{ }^{2}+\Delta^{2}}$, where $\epsilon_{p}=p-\mu$ and $\Delta$ is the superfluid gap. The field operator in Eq. (2) acts on a quasiparticle state $\left|p_{R}, p_{L}^{\prime}\right\rangle$ and restores it to the condensate. Conversely, it can also excite the condensate so that pair breaking and recombination occur simultaneously. We denote the matrix element for this process by $M_{q}$ and the neutrino matrix element by $M_{\nu}$. The time dependence on the field operator has been dropped, since the quasiparticle

\footnotetext{
${ }^{1}$ In this form, the quark field operator does not take into account the fact that diquarks may condense in preferred channels in color and flavor space, as, for instance in the three flavor color-flavor locked (CFL) phase, or the two flavor superconducting (2SC) phase (see, for example, [10]). We return later to comment on the expected modifications in "QCD condensed matter".
} 
lifetime is essentially infinite compared to the weak interaction time. Then, the expression for the emissivity is

$$
\begin{aligned}
\epsilon=\frac{1}{2} N_{\nu} N_{f} N_{c}\left(\frac{G_{F}}{\sqrt{2}}\right)^{2} \frac{(2 \pi)^{4}}{(2 \pi)^{12}} \int \frac{d^{3} p}{2 p_{0}} \frac{d^{3} p^{\prime}}{2 p_{0}^{\prime}} f\left(E_{p}\right) f\left(E_{p^{\prime}}\right) \\
\qquad \frac{d^{3} q_{1}}{2 q_{10}} \frac{d^{3} q_{2}}{2 q_{20}}\left(q_{10}+q_{20}\right)\left|M_{q}\right|^{2}\left|M_{\nu}\right|^{2} \delta^{4}\left(p+p^{\prime}-q_{1}-q_{2}\right),
\end{aligned}
$$

where $p$ and $p^{\prime}$ denote the four-momenta of quarks and $q_{1}$ and $q_{2}$ those of the neutrinos. The number of neutrino flavors is denoted by $N_{\nu}$. The simple factor of $N_{f} N_{c}$ outside arises, because we have disregarded strong interaction effects and flavor changing processes that occur at higher orders. The overall factor of $1 / 2$ avoids double counting of the same quasiparticle collision. $f$ denotes the FermiDirac distribution function. Summing over the neutrino spins yields the neutrino squared matrix element, $\left|M_{\nu}\right|^{2}=8\left(q_{2 \mu} q_{1 \nu}+q_{1 \mu} q_{2 \nu}-g_{\mu \nu} q_{1} \cdot q_{2}-i \epsilon_{\mu \nu \alpha \beta} q_{1 \alpha} q_{2 \beta}\right)$. The quark matrix element receives contributions from the vector $(V)$, axial $(A)$ and the mixed vector-axial $(M)$ channels.

The vector part of the interaction acting on the state $\left|p_{R}, p_{L}^{\prime}\right\rangle$ gives

$$
\left(\left|M_{q}\right|^{2}\right)_{V}^{\mu \nu}=c_{V}^{2}\left(u_{p} v_{p^{\prime}}+v_{p} u_{p^{\prime}}\right)^{2}\left(\bar{\chi}_{\overrightarrow{\mathbf{p}}, L} \gamma^{\mu} \chi_{\overrightarrow{\mathbf{p}}^{\prime}, L} \bar{\chi}_{\overrightarrow{\mathbf{p}}^{\prime}, L} \gamma^{\nu} \chi_{\overrightarrow{\mathbf{p}}, L}\right)
$$

where we have used the spinor identity $\bar{\chi}_{\overrightarrow{\mathbf{p}}, R} \gamma^{\alpha} \chi_{\overrightarrow{\mathbf{p}}^{\prime}, R}=\bar{\chi}_{\overrightarrow{\mathbf{p}}^{\prime}, L} \gamma^{\alpha} \chi_{\overrightarrow{\mathbf{p}}, L}$ \|11 in writing the term in the rightmost bracket. Using the standard normalization for massless spinors, the vector contribution to $\left|M_{q}\right|^{2}$ becomes

$$
\left(\left|M_{q}\right|^{2}\right)_{V}^{\mu \nu}=2\left(u_{p} v_{p^{\prime}}+v_{p} u_{p^{\prime}}\right)^{2} \cdot \frac{c_{V}^{2}}{4} \mathcal{T}
$$

where $\mathcal{T}=\operatorname{Tr}\left[\not p \gamma^{\mu}\left(1-\gamma_{5}\right) \not p^{\prime} \gamma^{\nu}\left(1-\gamma_{5}\right)\right]$. Utilizing the relation $\bar{\chi}_{\overrightarrow{\mathbf{p}}^{\prime}, R} \gamma^{\alpha} \gamma_{5} \chi_{\overrightarrow{\mathbf{p}}, R}=-\bar{\chi}_{\overrightarrow{\mathbf{p}}, L} \gamma^{\alpha} \gamma_{5} \chi_{\overrightarrow{\mathbf{p}}^{\prime}, L}=$ $\bar{\chi}_{\overrightarrow{\mathbf{p}}, L} \gamma^{\alpha} \chi_{\overrightarrow{\mathbf{p}}^{\prime}, L}$, the squared matrix elements for the axial and mixed contributions are found to be

$$
\left(\left|M_{q}\right|^{2}\right)_{A}^{\mu \nu}=2\left(u_{p} v_{p^{\prime}}-v_{p} u_{p^{\prime}}\right)^{2} \cdot \frac{c_{A}^{2}}{4} \mathcal{T} \quad \text { and } \quad\left(\left|M_{q}\right|^{2}\right)_{M}^{\mu \nu}=2\left(u_{p^{\prime}}^{2} v_{p}^{2}-v_{p^{\prime}}^{2} u_{p}^{2}\right) \cdot \frac{2 c_{V} c_{A}}{4} \mathcal{T}
$$

The total contribution from the quark squared matrix element is simply the sum $\left|M_{q}\right|^{2}=\left|M_{q}\right|_{V}^{2}+\left|M_{q}\right|_{A}^{2}+\left|M_{q}\right|_{M}^{2}$. The contraction of the neutrino and quark squared matrix elements yields, up to differences in couplings and coherence factors, a generic expression of the form

$$
\left|M_{q}\right|_{C}^{2} \times\left|M_{\nu}\right|^{2} \propto\left[16\left(q_{1} \cdot p\right)\left(q_{2} \cdot p^{\prime}\right)+48\left(q_{2} \cdot p\right)\left(q_{1} \cdot p^{\prime}\right)\right]
$$

where $C$ denotes any of the channels $V, A$, and $M$.

We turn now to the phase space integrations. The neutrino phase space integral is of the form

$$
\begin{aligned}
N_{\alpha \beta} & =\int \frac{d^{3} q_{1}}{2 q_{10}} \frac{d^{3} q_{2}}{2 q_{20}}\left(q_{1 \alpha} q_{2 \beta}+q_{2 \alpha} q_{1 \beta}\right)\left(q_{10}+q_{20}\right) \delta^{4}\left(q_{1}+q_{2}-Q\right) \\
& =\frac{\pi Q_{0}}{12}\left(Q^{2} g_{\alpha \beta}+2 Q_{\alpha} Q_{\beta}\right) \Theta\left(Q_{0}\right) \Theta\left(Q_{0}^{2}-|\overrightarrow{\mathbf{Q}}|^{2}\right),
\end{aligned}
$$

where an additional delta function in the total neutrino energy $Q_{0}$ and momentum $\overrightarrow{\mathbf{Q}}$ has been introduced. The obvious symmetry between $\alpha$ and $\beta$ in the above integral may be exploited to simplify the 
calculation (e.g. the $\epsilon_{\mu \nu \alpha \beta}$ terms from the neutrino squared matrix element vanish once the neutrino integral is performed). The momentum conserving delta function in Eq. (5) can be used to eliminate the integral over $\overrightarrow{\mathbf{p}}^{\prime}$. Next, we shift the $\overrightarrow{\mathbf{p}}$ integral using $\overrightarrow{\mathbf{p}} \rightarrow \overrightarrow{\mathbf{p}}+\frac{\overrightarrow{\mathbf{Q}}}{2}$, and in view of the restriction that $|\overrightarrow{\mathbf{Q}}| \leq Q_{0} \sim \Delta$ and $\Delta \ll|\overrightarrow{\mathbf{p}}| \sim \mu$, the energy conserving delta function becomes $\delta\left(Q_{0}-2 E_{p}\right)$. In addition, the two Fermi-Dirac functions both become $f\left(E_{p}\right)$. [

In the vector channel, the momentum shift $\overrightarrow{\mathbf{p}} \rightarrow \overrightarrow{\mathbf{p}}+\frac{\overrightarrow{\mathbf{Q}}}{2}$ allows us to write

$$
\left(u_{p} v_{Q-p}+v_{p} u_{Q-p}\right)^{2} \stackrel{\overrightarrow{\mathbf{p}} \rightarrow \overrightarrow{\mathbf{p}}+\frac{\vec{Q}}{2}}{\longrightarrow} u_{p+\frac{Q}{2}}^{2} v_{-p+\frac{Q}{2}}^{2}+v_{p+\frac{Q}{2}}^{2} u_{-p+\frac{Q}{2}}^{2}+2 u_{p+\frac{Q}{2}} v_{p+\frac{Q}{2}} v_{-p+\frac{Q}{2}} u_{-p+\frac{Q}{2}} .
$$

Using Eq. (四) and the expression for the quasiparticle energy, the first two terms become

$$
\left(\frac{1}{4} \frac{\Delta^{2}}{E_{p}^{2}}+\frac{x}{2} \frac{\Delta^{2}}{E_{p}^{2}} \frac{\epsilon_{p}}{E_{p}}+(x \rightarrow-x)\right)+O\left(x^{2}\right)
$$

where $x=\Delta / \mu \ll 1$, justifying the neglect of the $O\left(x^{2}\right)$ terms. The third term becomes $\Delta^{2} /\left(2 E_{p}^{2}\right)$. Thus, the coherence factors for the vector channel take the form

$$
\left(u_{p} v_{p^{\prime}}+v_{p} u_{p^{\prime}}\right)^{2}=\frac{\Delta^{2}}{E_{p}^{2}} .
$$

For the axial contribution to the quark matrix element, we retain terms up to $O\left(x^{2}\right)$ to get

$$
\left(u_{p} v_{Q-p}-v_{p} u_{Q-p}\right)^{2} \stackrel{\overrightarrow{\mathbf{p}} \rightarrow \overrightarrow{\mathbf{p}}+\frac{\overrightarrow{\mathbf{Q}}}{2}}{\longrightarrow}=\frac{(\overrightarrow{\mathbf{Q}} \cdot \hat{\mathbf{p}})^{2} \Delta^{2}}{4 E_{p}^{4}} .
$$

Eqs. (13) and (14) enable the coherence factor for the mixed contribution to be written as

$$
\left(u_{p^{\prime}}^{2} v_{p}{ }^{2}-v_{p^{\prime}}^{2} u_{p}{ }^{2}\right)=\sqrt{\frac{\Delta^{2}}{E_{p}^{2}} \frac{(\overrightarrow{\mathbf{Q}} \cdot \hat{\mathbf{p}})^{2} \Delta^{2}}{4 E_{p}^{4}}}=\frac{\overrightarrow{\mathbf{Q}} \cdot \hat{\mathbf{p}} \Delta^{2}}{2 E_{p}^{3}} .
$$

The mixed contribution, being odd in $\overrightarrow{\mathbf{Q}}$, vanishes on angular integration. Integrals over $\overrightarrow{\mathbf{Q}}$ can be performed using the formula

$$
\int d \Omega_{q} d|\overrightarrow{\mathbf{Q}}||\overrightarrow{\mathbf{Q}}|^{m}(|\overrightarrow{\mathbf{Q}} \cdot \overrightarrow{\mathbf{p}}|)^{n}=\frac{4 \pi}{n+1}|\overrightarrow{\mathbf{p}}|^{n} \int d|\overrightarrow{\mathbf{Q}}||\overrightarrow{\mathbf{Q}}|^{m+n} .
$$

The integrals over $Q_{0}, \overrightarrow{\mathbf{Q}}$, and $d \Omega_{p}$ are now straightforward, leaving only the integral over $|\overrightarrow{\mathbf{p}}|$. We can make a change of variable at the Fermi surface from $|\overrightarrow{\mathbf{p}}|$ to $E_{p}$ to cast the final result as a 1-dimensional integration over $E_{p}$. Collecting these results and summing the emissivities in the vector (V) and axial (A) channels, the $\nu \bar{\nu}$ emissivity from PBF in matter comprised of massless quarks is

\footnotetext{
${ }^{2}$ It is possible to calculate corrections resulting from $E_{p} \neq E_{p^{\prime}}$, but lead to more complicated expressions than found here (e.g, see Ref. [12,13]).
} 


$$
\epsilon^{R}(0)=N_{\nu}\left(\frac{G_{F} c_{V}}{\sqrt{2}}\right)^{2} \nu(0)\left(k_{B} T\right)^{7}\left(\frac{32}{15 \pi^{3}} F(y)\right)\left[1+\frac{1}{7}\left(\frac{c_{A}}{c_{V}}\right)^{2}\right],
$$

where $\nu(0)=N_{f} N_{c} \mu^{2} / \pi^{2}$ is the density of states at the Fermi surface $\mu$. The explicit form of the function $F(y)$ is

$$
F(y)=y^{2} \int_{y}^{\infty} d x \frac{x^{5}}{\sqrt{x^{2}-y^{2}}} \frac{1}{\left(e^{x}+1\right)^{2}}
$$

where $y=\beta \Delta=\Delta / k_{B} T$ and $x=E_{p} / k_{B} T$. The gap $\Delta$ depends on the temperature. In limiting situations, the temperature dependence of the superfluid gap $\Delta$ for ${ }^{1} \mathrm{~S}_{0}$ pairing is given by

$$
\begin{array}{rlr}
\Delta(T) & =\Delta(0)-\left(2 \pi k_{B} T \Delta(0)\right)^{1 / 2} \exp \left(-\frac{\Delta(0)}{k_{B} T}\right) \quad\left(T \ll T_{c}\right) \\
& =3.06 k_{B} T_{c}\left(1-T / T_{c}\right)^{1 / 2} \quad\left(T_{c}-T \ll T_{c}\right)
\end{array}
$$

with $\Delta(0)=1.76 k_{B} T_{c}$. In lieu of this, $F(y) \sim\left(1-\frac{T}{T_{c}}\right)$ as $T \rightarrow T_{c}^{-}$so that it vanishes quadratically with $\Delta$. For $T \ll T_{c}, F(y)$ vanishes exponentially as $\mathrm{e}^{-2 \Delta / T}$. Accurate fits of $F(y)$ valid over the entire range of $y$ may be found in Ref. [3].

It is instructive to compare the result Eq. (17) with that of Flowers et al. [1] for nonrelativistic neutrons:

$$
\epsilon=N_{\nu}\left(\frac{G_{F} c_{V}}{\sqrt{2}}\right)^{2} \nu(0)\left(k_{B} T\right)^{7}\left(\frac{32}{15 \pi^{3}} F(y)\right)\left[1+\frac{11}{42}\left(\frac{c_{A}}{c_{V}}\right)^{2}\left(\frac{v_{F_{n}}}{c}\right)^{2}\right]
$$

where $\nu(0)=m^{*} p_{F_{n}} / \pi^{2}$ is the density of states, $m^{*}$ is the Landau effective mass [5, and $v_{F_{n}}=p_{F_{n}} / m^{*}$ is the velocity at the Fermi surface. Since the PBF process occurs in the vicinity of the Fermi surface, the formal similarity of the results between the nonrelativistic and extreme relativistic cases is not surprising. The neutral current couplings (see Table 1), the density of states at the Fermi surface, and the results of phase space integrations are, however, somewhat different between the two cases. For both relativistic quarks and nonrelativistic neutrons, the dominating contribution arises from the vector channel although the suppression of the axial channel is more severe in the nonrelativistic case.

The main approximation made in the emissivity calculation for massless quarks is that $\Delta \ll \mu$. Modifications due to a finite quark mass $m$ enter in the dispersion relation $\epsilon_{p}=\sqrt{p^{2}+m^{2}}-\sqrt{p_{F}^{2}+m^{2}}$, with a corresponding density of states $\nu(0)=N_{f} N_{c} p_{F} \sqrt{p_{F}^{2}+m^{2}} / \pi^{2}$, and from corrections to the quark matrix element through the spinor normalization as $\Sigma_{s} u^{s}(p) \bar{u}^{s}(p)=\not p+m$. In what follows, we set the spin along the direction of the momentum of the massive quark. The quark field operator in the spin basis may be written as

$$
\psi(\overrightarrow{\mathbf{x}})=\sum_{\mathbf{p}}\left(e^{-i \overrightarrow{\mathbf{p}} \cdot \overrightarrow{\mathbf{x}}} u_{p}\left\{\chi_{\overrightarrow{\mathbf{p}},+} b_{\overrightarrow{\mathbf{p}},+}+\chi_{\overrightarrow{\mathbf{p}},-} b_{\overrightarrow{\mathbf{p}},-}\right\}+e^{i \overrightarrow{\mathbf{p}} \cdot \overrightarrow{\mathbf{x}}} v_{p}\left\{\chi_{\overrightarrow{\mathbf{p}},+} b_{\overrightarrow{\mathbf{p}},+}^{\dagger}-\chi_{\overrightarrow{\mathbf{p}},-} b_{\overrightarrow{\mathbf{p}},-}^{\dagger}\right\}\right)
$$

\footnotetext{
${ }^{3}$ Defined at the Fermi surface, $m^{*}$ accounts for momentum-dependent interactions in a potential model description. In a field-theoretical description, $m^{*}$ accounts for scalar interactions that off-set the bare mass.
} 
This acts on a quasiparticle state $\left|p_{+}, p_{-}^{\prime}\right\rangle$ and restores it to the condensate.

The vector channel squared matrix element is

$$
\begin{aligned}
\left(\left|M_{q}\right|^{2}\right)_{V}^{\mu \nu} & =2\left(u_{p} v_{p^{\prime}}+v_{p} u_{p^{\prime}}\right)^{2} \cdot c_{V}^{2} \operatorname{Tr}\left[M_{-}(p \prime) \gamma^{\mu} M_{-}(p) \gamma^{\nu}\right] \\
M_{-}(p) & =u_{-}(p) \bar{u}_{-}(p)=\frac{\not p+m}{2}\left(1-\gamma_{5} \not h(p)\right)
\end{aligned}
$$

with $n(q)=\left(\frac{|\overrightarrow{\mathbf{q}}|}{m}, \frac{q^{0}}{m} \hat{\mathbf{q}}\right)$. Setting $m=0$ reproduces the chirality projector that was used for the calculation in the massless case. Taking the nonrelativistic limit $m \gg|\overrightarrow{\mathbf{p}}|$, we find that only $\mu=0=\nu$ contributes to the matrix element. This is consistent with the nonrelativistic matrix elements considered in the neutron superfluid case [1].

The squared matrix element in the axial channel is

$$
\left(\left|M_{q}\right|^{2}\right)_{A}^{\mu \nu}=2\left(u_{p} v_{p^{\prime}}-v_{p} u_{p^{\prime}}\right)^{2} \cdot c_{A}^{2} \operatorname{Tr}\left[M_{-}(p \prime) \gamma^{\mu} \gamma_{5} M_{-}(p) \gamma^{\nu} \gamma_{5}\right]
$$

To perform the trace, notice that there are $\mathcal{O}\left(m^{2}\right), \mathcal{O}(m)$, and $\mathcal{O}\left(m^{0}\right)$ terms that may be grouped separately. The $\mathcal{O}(m)$ terms in the vector and axial channels vanish, because they involve the trace of an odd number of $\gamma$-matrices or are anti-symmetric in the very indices in which the overall neutrino integral is symmetric.

In the vector channel, the $\mathcal{O}\left(m^{2}\right)$ term is

$$
\frac{m^{2}}{4}\left\{4 g^{\mu \nu}+4 n^{\mu} n^{\prime \nu}+4 n^{\nu} n^{\prime \mu}-4\left(n . n^{\prime}\right) g^{\mu \nu}\right\},
$$

while the $\mathcal{O}\left(m^{0}\right)$ term is

$$
\frac{1}{4}\left\{4 p^{\mu} p^{\prime \nu}+4 p^{\nu} p^{\prime \mu}-4\left(p \cdot p^{\prime}\right) g^{\mu \nu}\right\}-\frac{1}{4} \operatorname{Tr}\left[\not p h \gamma^{\mu} \not p^{\prime} \not^{\prime} \gamma^{\nu}\right] .
$$

The axial channel gives similar contributions as above, but with the following changes: (i) the first and last terms in Eq. (24) are both of opposite signs, and (ii) the last term in Eq. (25) is of opposite sign.

Simple expressions for the emissivities that take into account quark masses may be obtained by retaining leading terms in some limiting situations.

(a) For $m / p_{F} \ll 1$, the projector in Eq. (22) reads $\frac{p}{2}\left(1+\gamma_{5}\right)+\frac{m}{2}$. In this case, the total emissivity is

$$
\epsilon^{R}(m)=\epsilon^{R}(0)\left[\left(1-\frac{m^{2}}{4 p_{F}^{2}}\right)+\frac{1}{7}\left(\frac{c_{A}}{c_{V}}\right)^{2}\left(1+\frac{7 m^{2}}{12 p_{F}^{2}}\right)\right],
$$

where $\epsilon^{R}(0)$ is the result in Eq. (17). In the context of the long-term cooling of neutron stars with quark cores, the utility of this result lies in the extent to which it applies for massive strange quarks.

(b) For $p_{F} / m \ll 1$, the projector in Eq. (22) becomes $m\left(1+\gamma_{0}\right)\left(1+\gamma_{5} \vec{\gamma} \cdot \hat{\mathbf{p}}\right)-|\overrightarrow{\mathbf{p}}|\left(\vec{\gamma} \cdot \hat{\mathbf{p}}-\gamma_{0} \gamma_{5}\right)$. In this case,

$$
\begin{aligned}
& \epsilon_{V}(m)=\epsilon_{V}(0)\left[\frac{m^{2}}{m^{2}+p_{F}^{2}}-\frac{31}{84} \frac{p_{F}^{2}}{m^{2}+p_{F}^{2}}\right] \text { and } \\
& \epsilon_{A}(m)=\left(\frac{c_{A}}{c_{V}}\right)^{2} \epsilon_{V}(0)\left[\left(\frac{v_{F}}{c}\right)^{2} \frac{11}{42}\left(\frac{m^{2}+p_{F}^{2} / 2}{m^{2}+p_{F}^{2}}\right)+\left(\frac{p_{F}^{2}}{m^{2}+p_{F}^{2}}\right)\right],
\end{aligned}
$$


where $\nu(0)$ and $F(y)$ in $\epsilon_{V}(0)$ involve the dispersion relation for massive particles. The changes from the extreme nonrelativistic result in Eq. (20) are easily understood. The factor $m^{2}+p_{F}^{2}$ in the denominator of the two terms inside the square bracket comes from energy factors in the Lorentz invariant phase space, while the numerator receives contributions from $\mathcal{O}\left(m^{2}\right)$ and $\mathcal{O}\left(m^{0}\right)$ terms in the trace. The heirarchy $\Delta \ll p_{F} \ll m$ should be maintained for these expressions to be valid. We can express the sum of the emissivities from the vector and axial channels in Eq. (27) as

$$
\epsilon^{N R}(m)=\epsilon_{V}(0)\left[1+\left(\frac{c_{A}}{c_{V}}\right)^{2}\left(\frac{v_{F}}{c}\right)^{2}\left\{\frac{11}{42}+\left(\frac{m^{*}}{m}\right)^{2}\right\}+\ldots\right]
$$

In this form, we recognize the nonrelativistic result of Flowers et al. in Eq. (20), and in the axial channel an additional term involving $\left(\mathrm{m}^{*} / \mathrm{m}\right)^{2}$, where $\mathrm{m}^{*}$ is the Landau effective mass (see footnote 2 ) defined through $p_{F}=m^{*} v_{F} / c=\left(\pi^{2} n_{B}\right)^{1 / 3}$. The ellipsis denotes terms that are smaller by further powers of $\left(p_{F} / m\right)^{2}$ compared to the preceding term in Eq. (28). The presence of the $\left(m^{*} / m\right)^{2}$ term and its importance in the case of superfluid protons for which $c_{V}$ is vanishingly small, was first noted in Ref. [12] and was obtained as a relativistic correction in PBF studies of a proton superfluid [12]. For further discussions on additional corrections, see Ref. [13].

The results in Eqs. (17) and (26) are the prinicipal new results of this work. For simplicity and for numerical comparison with emissivities from other processes in quark and baryonic matter, we will use the result in Eq. (17) in what follows to represent the $\nu \bar{\nu}$ emissivity from the PBF process in quark matter. In cgs units, Eq. (17) may be expressed as

$$
\epsilon_{q}^{\nu \bar{\nu}} \cong 1.4 \times 10^{20} N_{\nu} T_{9}^{7} F a_{q}\left(\frac{n_{B}}{n_{0}}\right)^{2 / 3} \mathrm{erg} \mathrm{cm}^{-3} \mathrm{~s}^{-1},
$$

where $N_{\nu}$ is the number of neutrino flavors, $T_{9}$ is the temperature in $10^{9}{ }^{\circ} \mathrm{K}, F$ is the result in Eq. (18), $a_{q}=c_{V}^{2}\left[1+\frac{1}{7}\left(\frac{c_{A}}{c_{V}}\right)^{2}\right]$ (see Table 1 ), and $n_{B}=p_{F_{q}}^{3} / \pi^{2}$ is the baryon density $\left(n_{0}=0.16 \mathrm{fm}^{-3}\right.$ is the empirical nuclear matter equilibrium density). This may be compared with the emissivities in normal quark matter from the bremsstrahlung $(q+q \rightarrow q+q+\nu+\bar{\nu})$ and modified Urca (e.g., $\left.d+d \rightarrow d+u+e^{-}+\bar{\nu}\right)$ processes [ [6]

$$
\begin{aligned}
\epsilon_{q}^{\text {brems }} & \simeq 3 \times 10^{19} N_{\nu} T_{9}^{8}\left(\frac{n_{B}}{n_{0}}\right)^{1 / 3} \mathrm{erg} \mathrm{cm}^{-3} \mathrm{~s}^{-1}, \\
\epsilon_{q}^{M U r c a} & \simeq \alpha_{c}^{2} \cos ^{2} \theta_{c} \epsilon_{q}^{\text {brems }},
\end{aligned}
$$

where $\alpha_{c}$ is the strong interaction coupling and $\theta_{C}$ is the Cabbibo angle. Note that both of these processes are suppressed by superfluidity by a factor of $\sim \exp \left(-2 \Delta / k_{B} T\right)$. Thus, as $T$ falls below $T_{c}$, the PBF process dominates the cooling in quark matter.

In baryonic matter, the $\nu \bar{\nu}$ emissivity from the PBF process is [1, 3]

$$
\epsilon_{B}^{\nu \bar{\nu}} \cong 1.65 \times 10^{21} N_{\nu} T_{9}^{7}\left(\frac{n_{B}}{n_{0}}\right)^{1 / 3}\left(\sum_{i} F_{i} a_{i} Y_{i}^{1 / 3} \frac{m_{i}^{*}}{m_{i}}\right) \operatorname{erg~cm}^{-3} \mathrm{~s}^{-1},
$$

where $a_{i} \cong c_{V i}^{2}$ and $Y_{i}=n_{i} / n_{B}$ is the baryon concentration of species $i$. The values of the neutral current hyperon couplings in Table 1, which were calculated in [14] by considering both the SU(3) singlet and octet contributions to the baryon current operators, suggest that in matter containing hyperons, the 
PBF process can occur with hyperons of all species. The values quoted in Table 1 of [13], where the $\Lambda$ and $\Sigma^{0}$ couplings were found to vanish and the $\Xi^{-}$coupling was very small, differ significantly from those in Table 1 probably due to the neglect of the singlet contributions in Ref. [13]. It is intriguing that at the same baryon density in baryonic and quark matter, $\epsilon_{B}^{\nu \bar{\nu}} / \epsilon_{q}^{\nu \bar{\nu}} \simeq$ (constant) $\cdot\left(n_{B} / n_{0}\right)^{-1 / 3}$ lies in the range $2-5$ for the densities of interest in the long-term cooling of solar mass compact stars.

The bremsstrahlung $(n+n \rightarrow n+n+\nu+\bar{\nu})$ and modified Urca process $\left(n+n \rightarrow n+p+e^{-}+\bar{\nu}\right)$ emissivities are [7], 8]

$$
\begin{aligned}
\epsilon_{B}^{\text {brems }} & \cong 4.4 \times 10^{19} N_{\nu} T_{9}^{8}\left(\frac{m_{n}^{*}}{m_{n}}\right)^{4}\left(\frac{n_{B}}{n_{0}}\right)^{1 / 3} R_{n n} \mathrm{erg} \mathrm{cm}^{-3} \mathrm{~s}^{-1} \\
\epsilon_{B}^{M U r c a} & \cong 10^{22}\left(Y_{e} \frac{n_{B}}{n_{0}}\right)^{1 / 3} \operatorname{erg~cm}{ }^{-3} \mathrm{~s}^{-1}
\end{aligned}
$$

where $R_{n n}$ is a density dependent reduction factor, that lies in the range $0.3-0.15$ for densities in the range $0.5-3 n_{0}$, recently computed in Ref. [8] utilizing on-shell $n n$ amplitudes (similar reductions are expected for the modified Urca process also).

So far, we have restricted ourselves to the case of isotropic ${ }^{1} \mathrm{~S}_{0}$ pairing, which provides a larger gap compared to anisotropic p-wave pairing $\left({ }^{3} \mathrm{P}_{2}\right.$ states). The fact that the smallest gap is the most relevant for long-term cooling highlights the importance of p-wave pairing though it requires higher energies (or densities) than s-wave pairing. For example, for neutrons in the lowest energy $m_{J}=0$ quantum number of the ${ }^{3} \mathrm{P}_{2}$ state, the anisotropy of the gap yields the relation [3]

$$
\Delta(T)=1.98 k_{B} T_{c}\left(1-T / T_{c}\right)^{1 / 2} \quad\left(T_{c}-T \ll T_{c}\right) .
$$

Just as for nucleons, the quark field operator in Eq. (21) must be modified to matrix-valued creation and annihilation operators due to the triplet nature of pairing. It can be obtained by a unitary transformation on the ground state [15] with the coherence factors in Eq. (田) acquiring additional factors from the angular dependence. The p-wave emissivity exhibits a $T$-dependence that is similar to that from s-wave pairing.

The effects of strong and electromagnetic correlations on weak interaction processes involving baryons are known to be large [16]. By considering only the electromagnetic correlations between protons and electrons, Leinson has recently demonstrated that $\nu \bar{\nu}$ emissivity from the PBF process from gapped protons is substantially larger than that obtained at the mean field level [17]. A similar enhancement may be expected in quark matter through a collective response of $u, d$, and $s$ quarks in a charge neutral environment even with miniscule concentrations of electrons.

Calculations to include effects germane to "QCD condensed matter" [10 would have to address modifications in the weak interaction hamiltonian that will likely lead to couplings and quasiparticle excitations that are modified from those considered here. We defer calculations of PBF processes in such phases and detailed cooling simulations of stars with quark matter to subsequent studies.

Research support from the DOE Grant No. FG02-88ER-40388 is gratefully acknowledged. We thank Dima Yakovlev for useful communications. 


\section{REFERENCES}

[1] E. Flowers, M. Ruderman, and P. Sutherland, Astrophys. J., 205, 541 (1976); see also D. Voskresensky and A. Senatorov, Sov. Phys.-JETP 63, 885 (1986).

[2] Ch. Schaab, D. Voskresensky, A.D. Sedrakian, F. Weber, and M.K. Weigel, Astron. Astrophys. 321, 591 (1997); D. Page, in The Many Faces of Neutron Stars, edited by R. Bucheri, J. van Paradijs, and M.A. Alpar (Kluwer Academic Publishers, Dordrecht, 1998), p. 539.

[3] D.G. Yakovlev, K. P. Levenfish and Yu A Shibanov, Physics-Uspekhi 42, 8 (1999); D.G. Yakovlev, A.D. Kaminker, O.Y. Gnedin, and P. Haensel, Phys. Rep. (2001), in press.

[4] D. Page, M. Prakash, J.M. Lattimer, and A.W. Steiner, Phys. Rev. Lett. 85, 2048 (2000).

[5] D. Bailin and A. Love, Phys. Rept. 107, 325 (1984); M. Alford, K. Rajagopal, and F. Wilczek, Phys. Lett. B422, 247 (1998); Nucl. Phys. B537, 443 (1999); B558, 219 (1999); R. Rapp, T. Schäfer, E. V. Shuryak, and M. Velkovsky, Phys. Rev. Lett., 81, 53 (1998); Annals Phys. 280, 35 (2000); D. T. Son, Phys. Rev. D59:094019 (1999); T. Schäfer and F. Wilczek, Phys. Lett. B 450, 325 (1999).

[6] N. Iwamoto, Phys. Rev. Lett. 44, 1637 (1980); Annals of Physics, Vol. 141, 1 (1982).

[7] B. Friman and O.V. Maxwell, Astrophys. J. 232, 541 (1979).

[8] C. Hanhart, D.R. Philips, and S. Reddy, Phys. Lett. B499, 9 (2001).

[9] S. Weinberg, Phys. Rev. Lett. 307, 178 (1976).

[10] K. Rajagopal and F. Wilczek, in Frontier of Particle Physics / Handbook of QCD, edited by M. Shifman, (World Scientific), to be published; hep-ph/0011333.

[11] C. Itzykson and J. B. Zuber, Quantum Field Theory, (McGraw Hill, New York, 1980).

[12] A. D. Kaminker, P. Haensel and D. G. Yakovlev, Astron. Astrophys. 345, L14 (1999).

[13] D. G. Yakovlev, A. D. Kaminker, and K. P. Levenfish, Astron. Astrophys. 343, 650 (1999).

[14] S. Reddy, M. Prakash, and J.M. Lattimer, Phys. Rev. D 58:013009 (1998); S. Reddy, Ph.D. Thesis (Stony Brook, 1998).

[15] R. Tamagaki, Prog. of Theor. Phys. 44, 905 (1970).

[16] A. Burrows and R.F. Sawyer, Phys. Rev. C58, 554 (1998), S. Reddy, M. Prakash, J.M. Lattimer, and J.A. Pons, Phys. Rev. C59, 2888, (1999).

[17] L. B. Leinson, Phys. Lett. B473, 318, (2000); Nucl. Phys. A687, 489 (2001). 
TABLE 1: The standard model neutral current vector and axial-vector couplings of neutrinos to quarks and baryons. The hyperon couplings are taken from Ref. [14] and include both the octet and singlet contributions. $\ell$ stands for $e-, \mu-$, or $\tau-$ type neutrinos. Numerical values are quoted using $\mathrm{D}=0.756, \mathrm{~F}=0.477$, and $\sin ^{2} \theta_{W}=0.23$.

\begin{tabular}{lcc}
\hline \hline Reaction & $c_{V}$ & $c_{A}$ \\
\hline$\nu_{\ell}+u \rightarrow \nu_{\ell}+u$ & $\frac{1}{2}-\frac{4}{3} \sin ^{2} \theta_{W}=0.1933$ & $\frac{1}{2}$ \\
$\nu_{\ell}+d \rightarrow \nu_{\ell}+d$ & $\frac{1}{2}+\frac{2}{3} \sin ^{2} \theta_{W}=0.6533$ & $-\frac{1}{2}$ \\
$\nu_{\ell}+s \rightarrow \nu_{\ell}+s$ & $\frac{1}{2}+\frac{2}{3} \sin ^{2} \theta_{W}=0.6533$ & $-\frac{1}{2}$ \\
\hline$\nu_{\ell}+n \rightarrow \nu_{\ell}+n$ & $-\frac{1}{2}$ & $-\frac{1}{2}(D+F)=-0.6165$ \\
$\nu_{\ell}+p \rightarrow \nu_{\ell}+p$ & $\frac{1}{2}-2 \sin ^{2} \theta_{W}=0.04$ & $\frac{1}{2}(D+F)=0.6165$ \\
$\nu_{\ell}+\Lambda \rightarrow \nu_{\ell}+\Lambda$ & $-\frac{1}{2}$ & $-\frac{1}{2}\left(F+\frac{D}{3}\right)=-0.3645$ \\
$\nu_{\ell}+\Sigma^{-} \rightarrow \nu_{\ell}+\Sigma^{-}$ & $-\frac{3}{2}+2 \sin ^{2} \theta_{W}=-1.04$ & $\frac{1}{2}(D-3 F)=-0.34$ \\
$\nu_{\ell}+\Sigma^{+} \rightarrow \nu_{\ell}+\Sigma^{+}$ & $\frac{1}{2}-2 \sin ^{2} \theta_{W}=0.04$ & $\frac{1}{2}(D+F)=0.6165$ \\
$\nu_{\ell}+\Sigma^{0} \rightarrow \nu_{\ell}+\Sigma^{0}$ & $-\frac{1}{2}$ & $\frac{1}{2}(D-F)=0.14$ \\
$\nu_{\ell}+\Xi^{-} \rightarrow \nu_{\ell}+\Xi^{-}$ & $-\frac{3}{2}+2 \sin ^{2} \theta_{W}=-1.04$ & $\frac{1}{2}(D-3 F)=-0.34$ \\
$\nu_{\ell}+\Xi^{0} \rightarrow \nu_{\ell}+\Xi^{0}$ & $-\frac{1}{2}$ & $-\frac{1}{2}(D+F)=-0.6165$ \\
\hline \hline
\end{tabular}

